Original Article

\title{
Effects of phenylpyruvate on the growth performance and intestinal microbiota in broiler chicken
}

\author{
Mir Zulqarnain Talpur, ${ }^{\mathrm{a}, 1}$, Wentong Peng, ${ }^{\mathrm{a}, 1}$, Yuxian Zenga, Peipei Xie ${ }^{\mathrm{a}}$, Jincheng Li ${ }^{\mathrm{a}}$, \\ Haijun Zhang ${ }^{\mathrm{b},}$ Gang Shu ${ }^{\mathrm{a}, *}$ and Qingyan Jiang, \\ ${ }^{a}$ Guangdong Laboratory of Lingnan Modern Agriculture, Guangdong Province Key \\ Laboratory of Animal Nutritional Regulation, College of Animal Science, South China \\ Agricultural University, Guangzhou China \\ ${ }^{\mathrm{b}}$ Key Laboratory of Feed Biotechnology of Ministry of Agriculture and Rural Affairs, Feed \\ Research Institute, Chinese Academy of Agricultural Sciences, Beijing, China \\ ${ }^{*}$ Corresponding authors \\ E-mail addresses: qingyan jiang, qyjiang@scau.edu.cn and Gang shu, shugang@scau.edu.cn \\ ${ }^{1}$ These authors contributed equally to this work
}

Simple Summary: The restriction on the use of antibiotic feed additives as growth promoters coerced researchers to investigate the potential utility of other alternatives. The purpose of this study was to see how dietary supplementation with phenylpyruvate affected broiler chicken growth, slaughter performance, gut health microbiota, and immunity. Results indicates that phenylpyruvate in the diet may positively modulate the cecal microbiota of broiler chickens without influencing the growth performance and improve carcass weight, intestinal morphology and some immunity related genes. This data can contribute to designing alternate approaches to replace antibiotics in modern poultry production and health.

\begin{abstract}
The purpose of this study was to see how dietary supplementation with phenylpyruvate affected broiler chicken growth, slaughter performance, gut health microbiota, and immunity. A total of 288 day old broiler chickens were randomly assigned to one of four groups (6 replicates each with 12 chicken). NC (basal diet), PC (basal diet plus antibiotic virginiamycin $15 \mathrm{ppm}$ ), LCP and HCP (basal diet plus phenylpyruvate $1 \mathrm{~kg} / \mathrm{t}$ and $2 \mathrm{~kg} / \mathrm{t}$, respectively). Results showed that PC had higher ADFI during the first 21 days, and better FCR than the NC, the LCP and HCP also improved broilers' FCR 0.001 and $0.037 \%$ in relation to $\mathrm{NC}$ respectively. $\mathrm{HCP}$ has a higher all-eviscerated ratio than $\mathrm{NC}$ and less abdominal fat than LCP. HCP has increased villus length and crypt depth in the ileum compared to the NC. Bursa was lower in HCP and thymus was lower in LCP and PC. In contrast HCP have lower proinflammatory cytokine IL-1, as well as lower TLR4. The phenylpyruvate improved family Selenomonadaceae, genus Megamonas Bacteroides species that are known for beneficial effects like for maintenance of the cell surface structure, regulating aromatic amino acids and C. jejuni-suppressive treatment respectively. Finally, phenylpyruvate feed supplement can be utilized to improve growth performance and positively modulate gut microbiota, however this is less efficient than antibiotics in improving growth performance, although more efficient in improving productive performance and gut morphology. Moreover, high dose of phenylpyruvate is more effective than low dose.
\end{abstract}

Keywords: phenylpyruvate; growth performance; intestinal morphology; immunity; cecal microbiota. 


\section{Introduction}

The use of antibiotic feed additives as growth promoters are banned. Because of the growing threat of antibiotic resistance, consumers are increasingly concerned about drug residues in meat products [1]. As an outcome, antibiotic-free poultry meat is becoming increasingly popular around the world, not only due to consumers demand, but also the support of entire breeding industry and the voluntarily stopped antibiotics use in broiler production [2]. However, there are numerous obstacles, as the use of AGPs has declined, this has resulted in increased poultry performance issues, increased feed conversion ratio (FCR), and increased the occurrence of certain infectious diseases [3]. This scenario has compelled researchers to investigate the feasibility of alternative poultry production methods. Amino acids have recently received attention in poultry science due to the nutritional and healthier properties of feed ingredients [4].

Amino acids and their metabolites has a beneficial effect because it eliminates the negative effects of antibiotics, which resulted in the elimination of the intestinal microbiota without distinguishing between the harmful and beneficial microbiota [5,6]. Amino acids and their metabolic intermediates are critical components of animal growth and development [7]. In general, poultry receives nutrients and amino acids through the consumption of natural feedstuffs, but some key essential amino acids, such as Phe, can only be obtained through diet [8]. Phe is required for the synthesis of thyroid hormones, which control metabolic processes and thus influence the growth of various body structures, feed efficiency, oxygen consumption, protein synthesis and metabolism, thermogenesis, and acclimation to environmental changes [9]. In mammals, a Phe-rich diet reduces the effects of an acute stressor [10]. Cotoia, et al. [11] demonstrated that hydroxyphenyl pyruvate, a Phe metabolite, improves cellular survival under stress conditions in rats. This amino acid is metabolized via two routes: oxidation to Tyr and transamination to phenyl pyruvate, both of which are part of the classic metabolic pathways [12]. In addition, Phenylpyruvate can be catabolized to hydroxyphenyl pyruvate, becoming part of the energy metabolism. Some studies have been found that human body can convert phenylpyruvate into the corresponding essential amino acids phenylalnine because have the same carbon skeleton as the corresponding amino acids, and in vivo, they are transaminated by various tissues, including jejunum, liver, and muscle. Nonetheless, the effects of AAsupplemented diets, such as phenylalanine (Phe), on any animal species have received little attention.

Hence, they provide amino acids for protein synthesis without the introduction of additional nitrogen. Thus, supplements containing AAs phenylalanine or their metabolite phenylpyruvate have to be given in order to prevent protein $(\mathrm{PR})$ malnutrition offers multiple advantages in that they provide reductions in both dietary crude protein concentrations and the excretion of dietary nitrogen into the environment. However, there is no previous work on the metabolism of this amino acids in our objective species. Although functional requirements for this amino acid clearly seems to maximize growth performance and carcass characteristics of fast-growing broilers.

We hypothesized that phenylpyryate will improve growth and production performance and immunity linked to nutrient digestibility and gastrointestinal ecology in broilers fed corn soybean meal diet with phenylpyryate. Thus, the objective of this study was to evaluate the potential of adding phenylpyruvate to the diets of broilers to function as an alternative to antibiotic growth promoters and determine the effect of supplement on body, with primary response outcomes including live performance productive yield and immune response. Specifically, we evaluated the effects of phenylpyruvate on growth performance, intestinal morphology, immunity and cecal microbiota. 


\section{Materials and methods}

South China Agricultural University's Animal Care and Use Committee reviewed and approved the protocol (ACE-CAAS-20201211). All procedures were carried out in accordance with the guidelines in the Ministry of Science and Technology's Guide for Experimental Animals (Guangzhou, China), and every effort was made to minimize suffering.

\subsection{Birds, Diets, and Experimental Design}

The broiler chicks were managed in accordance with Arbor Acre broiler chicken guidelines. phenylpyruvate was purchased from Shanghai Aladdin Bio-Chem Technology Co.,LTD. The test diet was formulated concerning NRC [13] and NY/T 33-2004 and combined with the AA broiler feeding manual. Four groups of 288-day-old broiler chickens were divided at random (6 replicates in each group each with 12 chicken). Negative control (NC; basal diet), Positive control (PC; basal diet +antibiotic virginiamycin $15 \mathrm{ppm}$ ) one of the 2 phenylpyruvate feed additives diets prepared by adding, phenylpyruvate additive low dose (LCP; basal diet $+1 \mathrm{~kg} / \mathrm{t}$ phenylpyruvate feed additive) and phenylpyruvate feed additive high dose (HCP; $2 \mathrm{~kg} / \mathrm{t}$ phenylpyruvate feed additive). Broiler chicks were fed and watered ad libitum, and a 243L:1D light regime was maintained. The experiment lasted 42 days, divided into a starter period ( 0 to $21 \mathrm{D})$ and a finisher period (22 to $42 \mathrm{D})$. The specific experimental design is shown in Table 1. The composition and nutrient content of the basic diet are shown in Table 2 . The composition of the basal diet is shown in Table 2. Dietary treatments consisted of the basal diet supplemented with 0 (NC; control), Positive control (PC; basal diet +antibiotic virginiamycin $15 \mathrm{ppm}$ ) $1 \mathrm{~kg} / \mathrm{t}$ phenylpyruvate (LCP), $2 \mathrm{~kg} / \mathrm{t}$ phenylpyruvate (HCP) The experimental feed was pelletized and fed in the form of pellets.

\section{Table 1}

Experimental design

\begin{tabular}{lll}
\hline No. & Group & Add level \\
\hline $1 \#$ & Negative control (NC) & Basic diet + no antibiotics \\
$2 \#$ & Positive control (Antibiotic) (PC) & Basic diet + antibiotics (antibiotic at $15 \mathrm{ppm})$ \\
$3 \#$ & (LCP) phenylpyruvate feed (Low dose) & Basal diet + phenylpyruvate $(1 \mathrm{~kg} / \mathrm{t})$ \\
$4 \#$ & (HCP) phenylpyruvate feed (High dose) & Basal diet + phenylpyruvate $(2 \mathrm{~kg} / \mathrm{t})$ \\
\hline
\end{tabular}

\section{Table 2}

Test diet formula and nutritional level

\begin{tabular}{ccc}
\hline Items & Starter diet & Grower diet \\
\hline Ingredient $\%$ & $0 \sim 21 \mathrm{~d}$ & $21 \sim 42 \mathrm{~d}$ \\
Corn & 56.59 & 59.96 \\
Soybean meal & 25.95 & 20 \\
Cotton meal & 4.5 & 4.42 \\
Wheat meal & 4 & 5 \\
Wheat middlings & 2 & 2 \\
Oil & 2.49 & 4.5 \\
Calcium hydrogen phosphate & 1.82 & 1.58 \\
Mountain flour & 1.35 & 1.27 \\
Salt & 0.35 & 0.35 \\
Lysine & 0.35 & 0.35 \\
Methionine & 0.23 & 0.21 \\
Threonine & 0.05 & 0.04 \\
Premix of trace elements ${ }^{(1)}$ & 0.2 & 0.2 \\
Vitamin premix ${ }^{2}$ & 0.02 & 0.02
\end{tabular}




\begin{tabular}{ccc}
\hline Choline chloride & & 0.1 \\
Total & 0.1 & 100 \\
Nutrition level (3) & 100 & 3160 \\
Metabolizable energy (kcal/kg) & & 19.95 \\
Crude protein (\%) & 2980 & 0.9 \\
Calcium (\%) & 21.95 & 0.4 \\
Available phosphorus (\%) & 1 & 1.15 \\
Lysine (\%) & 0.45 & 0.54 \\
Methionine (\%) & 1.30 & 0.87 \\
Methionine+Cystine (\%) & 0.58 & 0.75 \\
Threonine (\%) & 0.94 & 0.2 \\
Tryptophan (\%) & 0.84 & 0.23 \\
\hline
\end{tabular}

Note: (1) Premix of trace elements (provides milligrams per kilogram of feed): $\mathrm{Cu} 8, \mathrm{Zn} \mathrm{75,} \mathrm{Fe} \mathrm{80,} \mathrm{Mn}$ 100 , Se 0.15 , I 0.35

(2)Vitamin premix (provided per kilogram of feed): Vitamin A 12500 IU, Vitamin D3 2500 IU; The following are mg: Vitamin E 18.75, Vitamin K3 2.65, Vitamin B12, Vitamin B2 6, Vitamin B12 0.025 , Biotin 0.0325 , Folic acid 1.25, pantothenic acid 12, niacin 50

(3) the results are calculated values

\subsection{Growth Performance}

During the experiment, mortality was recorded daily. On the $21^{\text {st }}$ and $42^{\text {nd }} \mathrm{D}$, body weight (BW) and feed intake were recorded based on the pen. The average daily feed intake (ADFI), average daily gain (ADG), and feed conversion rate (feed conversion ratio, FCR) were calculated.

\subsection{Sampling Procedure}

On the $21^{\text {st }}$ and $42^{\text {nd }} \mathrm{D}$, one chicken was randomly selected from each replication, to obtain serum samples, blood samples were collected and centrifuged, which were then stored at $-20^{\circ} \mathrm{C}$ for further study. Bodyweight was recorded after a $12 \mathrm{~h}$ feed withdrawal of one chicken with BW close to the mean picked from each replicate from each group and sacrificed for the measurements on the $42^{\text {nd }} \mathrm{D}$ after blood sampling.

After bleeding and defeathering, full eviscerated weight was measured when all the internal organs were excised (head, neck, claws, trachea, esophagus, stomach, gizzard, gizzard contents, intestines, spleen, pancreas heart, liver, glandular, abdominal fat, full craw and cutin membrane, and gonads). At 30 minutes postmortem, the whole thigh and breast muscle tissue were removed from the animal carcasses. The abdominal fat, thigh muscle and Breast muscle (including all of fat visceral region and abdominal cavity) were weighed, as a percentage of body weight, the weight percentage of fully eviscerated yield was calculated. Breast muscle, thigh muscle, abdominal fat, and liver weight percentages were calculated as a percentage of the weight of the eviscerated carcass.

The thymus, bursa, and spleen were immediately removed, dried, and individually weighed $(\mathrm{g})$ for each individual, and the ratio of thymus, bursa, and spleen weight: body weight $(\%)$ was calculated, where Organ index $=$ (organ weight in grams) / (live weight, in grams) $\mathrm{x}$ 100. For the expression analysis of inflammatory factors middle section of the jejunum and ileum tissue were placed in a $2 \mathrm{~mL}$ centrifuge tube, and then quickly frozen in liquid nitrogen and transferred to an ultra-low temperature refrigerator at $-80^{\circ} \mathrm{C}$. For intestinal morphology, the duodenum, jejunum, and ileum were collected $2 \mathrm{~cm}$ each and placed in a $10 \%$ middle $(\mathrm{PH}=7.4)$ fixed in formalin fixative and stored in a refrigerator at $4^{\circ} \mathrm{C}$. Cecal contents were placed in a $1.5 \mathrm{~mL}$ centrifuge tube, quickly frozen in liquid nitrogen, and then transferred to an ultra-low temperature refrigerator at $-80^{\circ} \mathrm{C}$ for storage. 


\subsection{Inflammatory Factors in the Intestine}

\subsubsection{RNA isolation}

Chicken intestinal tissues were collected, and RNA was extracted according to the manufacturer's instructions. RNA was then purified using the RNEasy Mini kit (QIAGEN), where tissues were homogenized in $600 \mathrm{ml}$ of RLT buffer With Tissue Ruptor that is a handheld rotor-stator homogenizer (Qiagen Inc.), as well as total RNA was eluted in $50 \mu 1$ of DNase-free water before being stored at $-80^{\circ} \mathrm{C}$. A spectrophotometer was used to measure the amount of RNA (NanoDrop Products, Wilmington, DE). Each sample's total RNA (300 ng) was extracted. The Primer Express software program was used to design the probe and primer set for the 28S rRNA and cytokines (PE Applied Biosystems, Foster City, CA). A welldesignated technique was used to quantify chemokine and cytokine mRNA expression. Probes and primers for chemokines and cytokines are listed in Table 3. One-step RT-PCR master mix reagents and TaqMan fast universal PCR master mix were used for the qRT-PCR. The Applied Biosystems 7500 Fast real-time PCR system was used for amplification and detection of specific products, with the following cycle profile: one cycle of $48^{\circ} \mathrm{C}$ for $30 \mathrm{~min}$ and $95^{\circ} \mathrm{C}$ for $20 \mathrm{~s}$ followed by 40 cycles of $95^{\circ} \mathrm{C}$ for $3 \mathrm{~s}$ and $60^{\circ} \mathrm{C}$ for $30 \mathrm{~s}$. The enhanced fluorescence found by the 7500 Fast sequence detection system during PCR amplification was due to hydrolysis of the target-specific probes by the rTth DNA polymerase's 5' nuclease activity. Normalization was carried out on the housekeeping gene $28 \mathrm{~S}$ rRNA. For each sample, the correction factor was calculated by dividing the mean threshold cycle (CT) value for the $28 \mathrm{~S}$ rRNA-specific product in that sample by the overall mean $\mathrm{CT}$ value for the $28 \mathrm{~S}$ rRNA-specific product in all samples. The following is how the corrected cytokine mean was calculated: average of each replicate $\times$ cytokine slope $/ 28 \mathrm{~S}$ slope $\times 28 \mathrm{~S}$ correction factor.

\section{Table 3}

Real-time quantitative RT-PCR probes and primers

\begin{tabular}{lll}
\hline Gene & Sequence type-Probe/primer sequence & Accession number \\
\hline IL-1 $\beta$ & $\begin{array}{l}\text { forward 5'--TTCATTACCGTCCCGTTG-3' } \\
\text { reverse 5'--GCTTTTATTTCTCCAGTCACA-3' } \\
\text { forward 5'--AAATCCCTCCTCGCCAATCT-3 } \\
\text { IL-6 }\end{array}$ & (NM_204524.1) \\
IL-10 & $\begin{array}{l}\text { forward 5'--CGCTGTCACCGCTTCTTCA-3 } \\
\text { reverse 5'--TCCCGTTCTCATCCATCTTCTC-3 } \\
\text { forward 5'--GTGCCCACGCTGTGCTTAC -3 } \\
\text { IL-4 }\end{array}$ & (NM_204628.1) \\
IFN $\gamma$ & $\begin{array}{l}\text { forward 5'--GCCCTTCCTGTAACCAGATG-3 } \\
\text { reverse 5'--ACACGACAGCCAAGTCAACG-3 } \\
\text { forward 5'--TTTCCAAGCACCAGATAGCAACA-3 } \\
\text { TLR4 }\end{array}$ & (NM_001004414.2) \\
& reverse 5'--TTCCAGCACAAGCCCTGAAATTA-3 & (NM_001007079.1) \\
\hline
\end{tabular}

For the genomic DNA sequence.

Interleukin 1 beta, Toll-like receptor 4, Interleukin 6, Interleukin 10, Interleukin 4, Interferon gamma.

\subsection{Intestinal Morphology}

For intestinal morphology collected duodenum, jejunum, and ileum samples were placed in a $10 \%$ middle $(\mathrm{PH}=7.4)$ fixed in formalin fixative prepared in paraffin sections. Perform HE staining, observe under an optical microscope, randomly select 5 discontinuous fields for each slice, and count 3 sets of data for each field. The height of the villi refers to the length from the top of the villi to the base of the villi, and the depth of the crypt refers to the 
distance from the bottom of the intestinal gland to the base opening between the two villi. Measure villus height (VH), crypt depth (CD), and calculate villus height/crypt depth (VH/CD).

\subsection{Cecal Microflora}

\subsubsection{DNA Extraction}

Dewar, et al. [14] as previously described, using a QIAamp Fast DNA stool mini kit (Qiagen), microbial DNA was extracted from cecum samples as per manufacturer's guidelines. The quality and the quantity of harvested DNA were measured using a NanoDrop 2000 UVvis spectrophotometer (Thermo Scientific, Wilmington, USA); additionally, $1 \%$ agarose gel electrophoresis was used to ensure DNA quality. 338 F (5--ACTCCTACGGGAGGCAGCA3) and 806 R (5--GGACTACHVGGGTWTCTAAT-3) were used to amplify the V3-V4 hypervariable regions of the bacterial 16S rRNA gene. A thermocycler PCR system (Gene Amp 9700, ABI, USA) was used to conduct PCR and the reactions were performed in triplicates: $20 \mu \mathrm{L}$ mixture, containing $4 \mu \mathrm{L}$ of $5^{\circ} \varnothing$ FastPfu Buffer, $2 \mu \mathrm{L}$ of $2.5 \mathrm{mmol} / \mathrm{L}$ dNTPs, $0.8 \mu \mathrm{L}$ of each primer $(5 \mu \mathrm{mol} / \mathrm{L}), 0.4 \mu \mathrm{L}$ of FastPfu Polymerase, and $10 \mathrm{ng}$ of template DNA. To purify the extracted PCR products from a $2 \%$ agarose gel, the AxyPrep DNA Gel Extraction Kit (Axygen Biosciences, Union City, CA, USA) was used, which were then quantified using QuantiFluor-ST (Promega, USA) according to the manufacturer's guidelines.

\subsubsection{Pyrosequencing and Bacterial Data Processing}

According to Majorbio Bio-Pharm Technology Co. Ltd, Shanghai, China, purified amplicons were pooled and sequenced in equimolar concentrations on an Illumina MiSeq PE300 platform (Illumina, San Diego, USA). The sequencing results were subjected to bioinformatics analysis. Quality-filtered by Trimmomatic and FLASH software with the following criteria were the established raw fastq sequences: (i) bases with a score of less than $<20$ were excluded, and at any site with an average quality, the reads were truncatedscore < 20 across a 50-bp sliding window; (ii) Trimmomatic software was used to delete truncated reads that were less than $50 \mathrm{bp}$ in length; (iii) reads of up to 2 mismatched nucleotides and more than 0 barcode mismatches were discarded; (iv) using the FLASH programme, the merged reads were removed; additionally, sequences that overlapped longer than $10 \mathrm{bp}$ were assembled using their overlap sequences; (v) readings that have not been assembled have been excluded. After this, high-quality sequences acquired were paired with samples based on their barcodes, clustering these reads with up to 97 percent similarities in operational taxonomic units using U search (version 7.1). Then, using the Ribosomal Database Project classifier algorithm, the chimaera and singletons were eliminated and allocated to taxa. A BLAST search for taxonomic classification was performed using QIIME at the 70 percent confidence level in the SILVA database (version 1.8.0). The taxonomic composition was investigated at the phylum, family, class, genus, and species levels using Vegan packages in R. Principal coordinate analysis of the complete diversity of microbial communities based on species levels based on Bray-Curtis distance was managed by R; [15].

\subsection{Statistical Analysis}

Data regarding growth performance, production performance, haematological parameters, carcass, and immunological parameters were analysed using SPSS software (IBM Corp. IBM SPSS Statistics for Windows, Version 23.0. Armonk, NY, USA). GraphPad Prism 7.0 software (Chicago, IL, USA). The data are presented as mean \pm SEM. $P \leq 0.05$ was considered to be 
statistically significant. Prior to variance analysis, the data on broiler mortality were transformed using arc sine (angular) transformation values to achieve a more normal distribution of the data [16].

\section{Results}

\subsection{Growth performance}

Bacterial resistance to antibiotics used on animals is a public health concern. Throughout the feeding trial period, all of the chickens in our study were healthy. During the first 21 days, The LCP and HCP groups were not change ADFI in relation to the control group, and presented lower results compared to the $\mathrm{PC}$ group $(\mathrm{P}<0.05$; Figure 1$)$, while the ADG and FCR were showed no significant differences $(P>0.05$; Figure 1$)$. There will be no significant differences were found in ADG, ADFI, or FCR over the next 21 days. However, over the entire 42-days birth-to-shelf process, the PC group had better FCR than the NC group $(P<0.05)$, while the LCP group and HCP group also improved broilers' FCR 0.001 and $0.037 \%$ in relation to $\mathrm{NC}$ group $(P<0.01)$, although higher than $\mathrm{PC}$ group $(\mathrm{P}<0.05$; Figure $1 \mathrm{C})$.
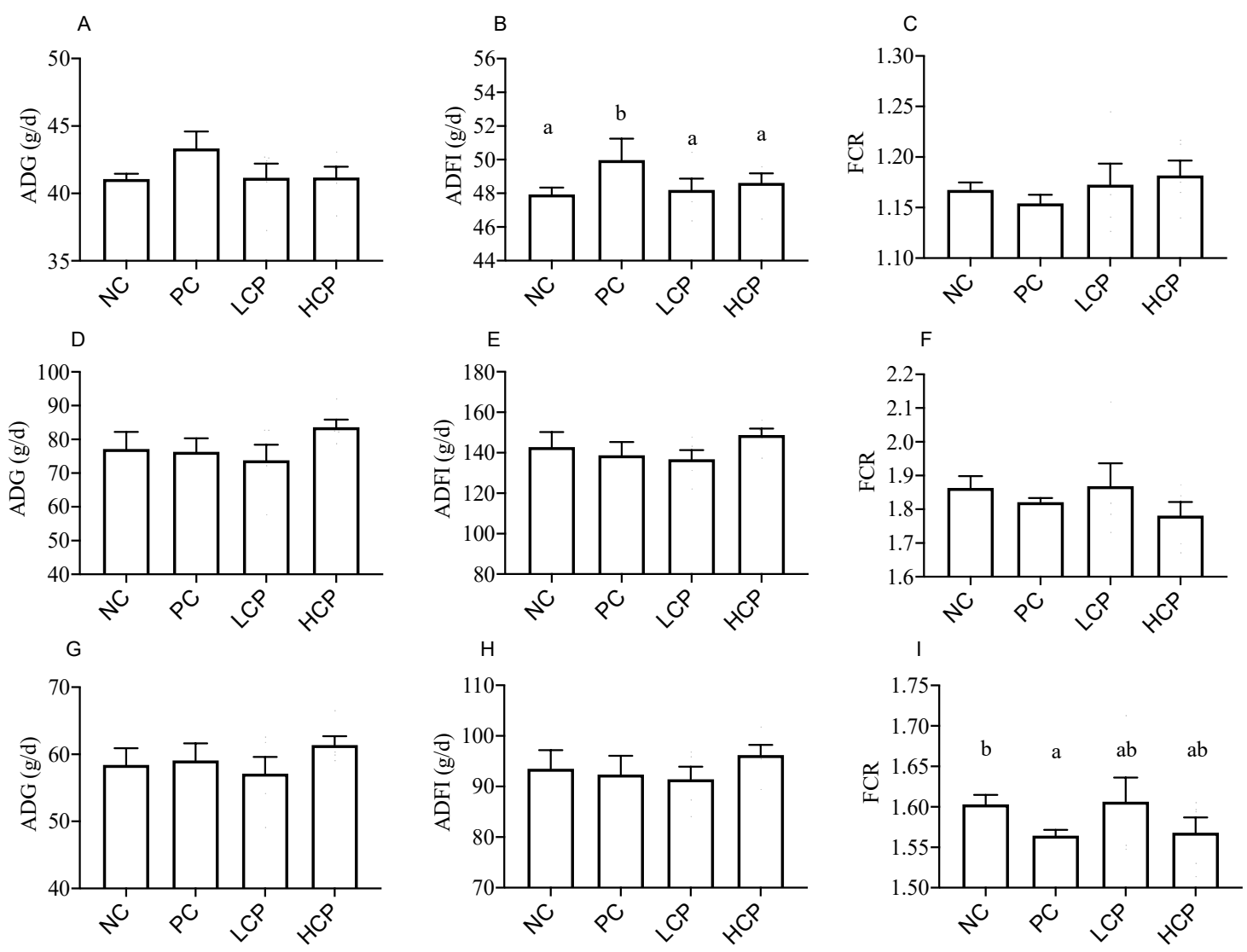

Figure 1. Effect of phenylpyruvate supplementation and antibiotic on growth performance in broiler chicken.

Negative control (NC), Positive control (Antibiotic) (PC), phenylpyruvate (low dose) (LCP), phenylpyruvate (high dose) (HCP). A, B, C, 1-21d, D, E, F, 21-42d, G, H, I, 42D.

ADG, average daily gain; ADFI, average daily feed intake; FCR (feed: gain = g: g), feed conversion ratio; BW, body weight.

Note : Values with different superscripts in the same row differ $(P<0.05)$. 
Mortality was not significantly different at any time period $(\mathrm{P}>0.05$; Table 4$)$, however $1-21$ days mortality was higher in the NC and PC group, although in LCP and HCP groups were recorded no mortality, whereas for 22-42 days mortality was increased and overall, 1-42 days mortality was higher in $\mathrm{HCP}$ group and similar with the NC group.

Table 4

Mortality rate

\begin{tabular}{|l|l|l|l|l|}
\hline Mortality rate & NC & PC & LCP & HCP \\
\hline $1-21$ day & $1.19 \pm 1.19$ & $1.19 \pm 1.19$ & 0 & 0 \\
\hline $22-42$ day & $6.06 \pm 2.40$ & $4.76 \pm 1.68$ & $5.00 \pm 2.04$ & $8.33 \pm 2.64$ \\
\hline $1-42$ day & $7.25 \pm 2.90$ & $5.95 \pm 1.54$ & $5.00 \pm 2.04$ & $8.33 \pm 2.64$ \\
\hline
\end{tabular}

Values transformed by arc sine (angular) transformation

Negative control (NC), Positive control (Antibiotic) (PC), phenylpyruvate (low dose) (LCP), phenylpyruvate (high dose) (HCP).

Note : Values with different superscripts in the same row differ $(P<0.05)$.

\subsection{Duodenum, jejunum, and ileum morphology}

In the ileum, villus length was greater in the HCP group than the NC group (P 0.05: Figure 2), whereas crypt depth was greater in the HCP group than the $\mathrm{PC}$ group $(\mathrm{P}<0.05)$. However, in the duodenum and jejunum, villus height, crypt depth, and the $\mathrm{VH} / \mathrm{CR}$ ratio in the duodenum, jejunum, and ileum were all comparable across groups.
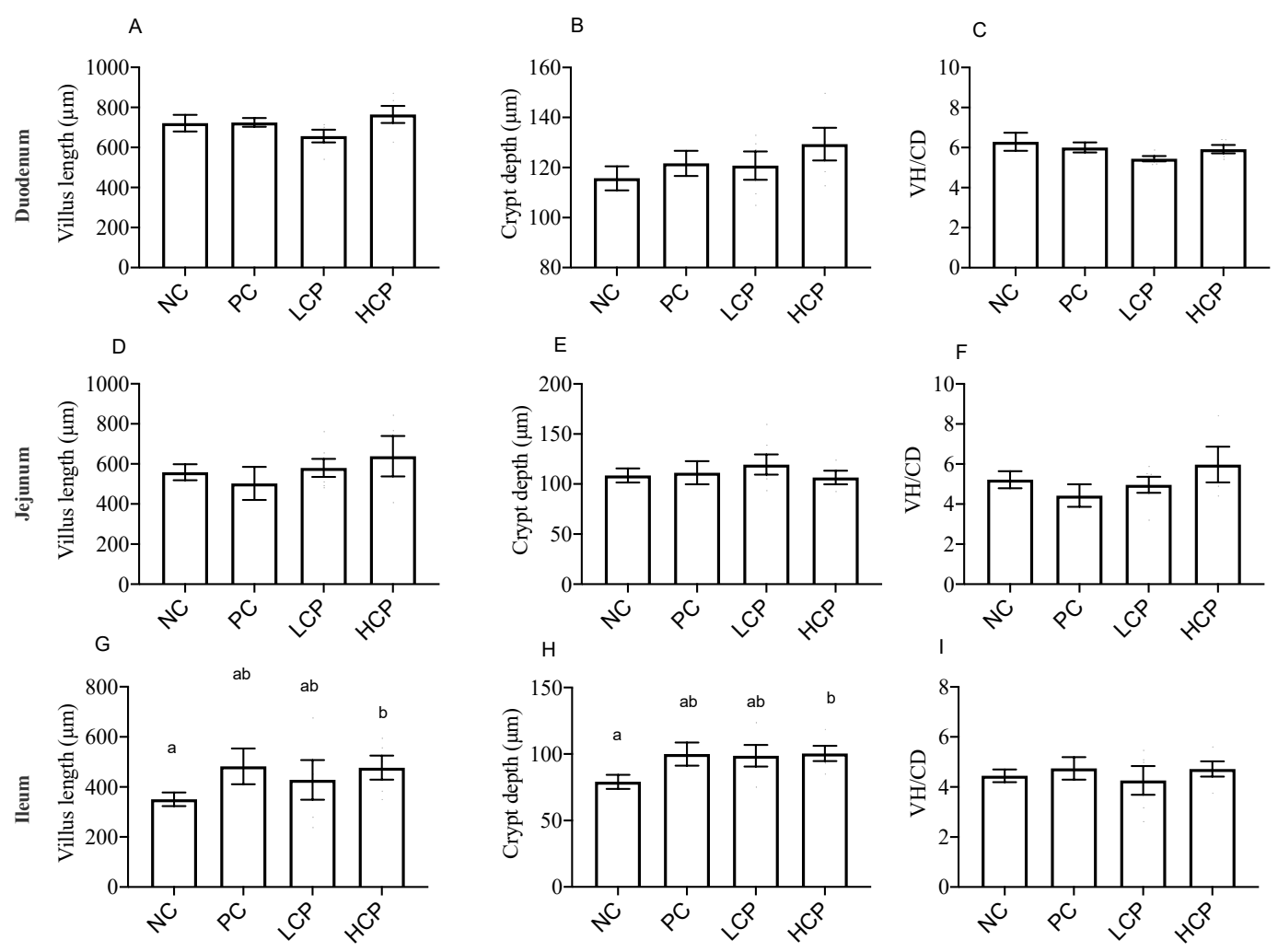

Figure 2. Effect of antibiotic and phenylpyruvate on duodenum, jejunum, and ileum in broiler chicken

Negative control (NC), Positive control (Antibiotic) (PC), phenylpyruvate (low dose) (FFL), phenylpyruvate (high dose) (FFH). 
Values with different superscripts in the same row differ $(P<0.05)$. Different color bar plots showing different groups.

\subsection{The digestive tract's morphometric characteristics}

In the current study, diet supplementation with phenylpyruvate significantly affected the morphometric traits of the digestive tract; the intestine of all groups showed normal tissue architecture with increased intestinal villi in the LCP and HCP group and crypt depth in the PC group (Figure 3).

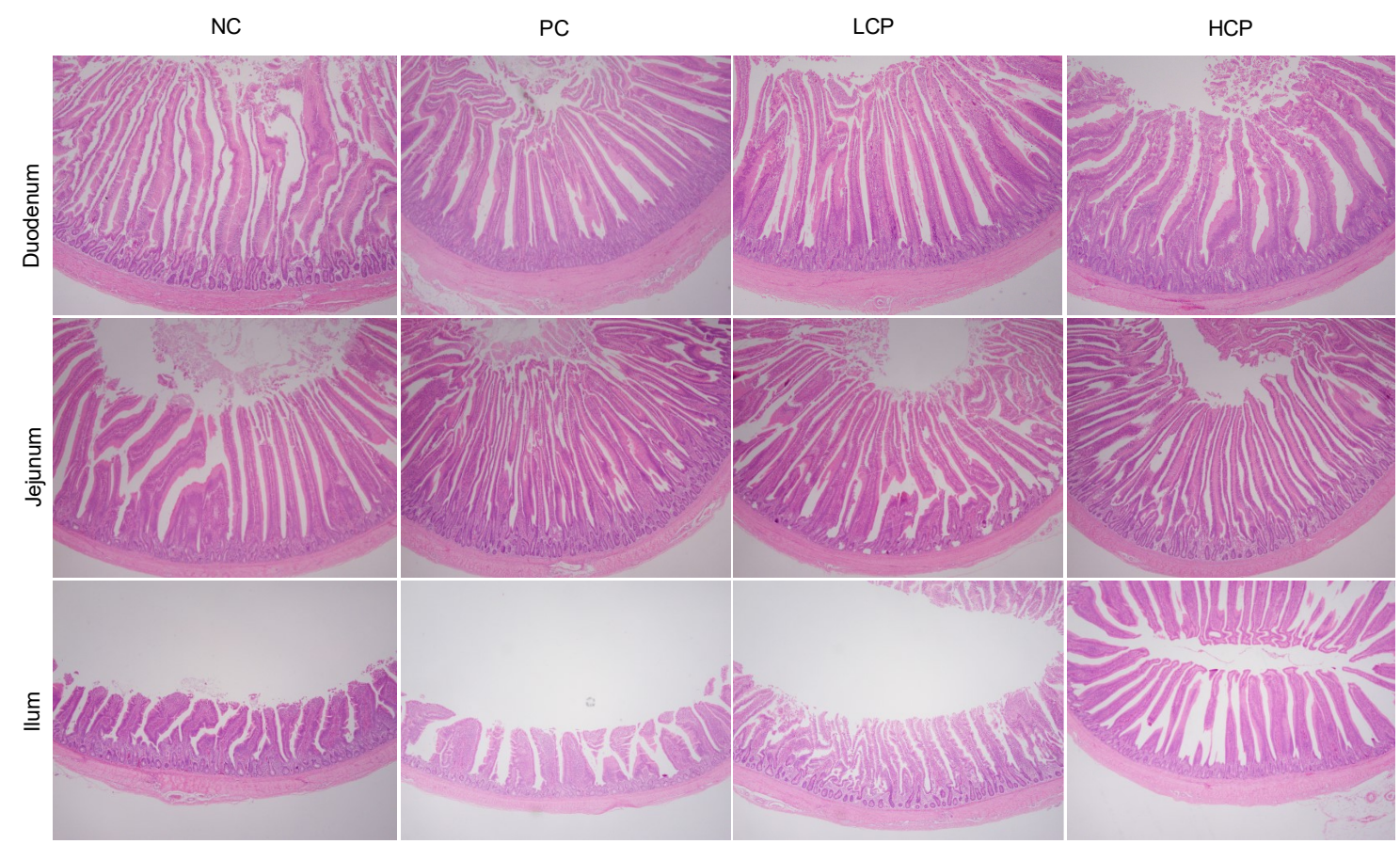

Figure 3. Effect of antibiotic and and phenylpyruvate on duodenum, jejunum and ileum's morphology in broiler chicken.

Negative control (NC), Positive control (Antibiotic) (PC), phenylpyruvate (low dose) (LCP), phenylpyruvate (high dose) (HCP).

The gross and microscopic views of different parts of chickens' intestine in treatment groups and control group.

The intestine parts duodenum, jejunum, and ileum sections were used. Histology was assessed for intestine sections embedded in paraffin - sections stained with hematoxylin and eosin.

\subsection{Slaughter Performance}

HCP had a higher all eviscerated ratio than the NC group, while HCP had a lower abdominal fat ratio than LCP group $(\mathrm{P}<0.05)$. However, on the other hand, the ratio of breast and thigh muscles, as well as the relative rate of liver, were evaluated, but no differences were found between groups (Figure 4). Whereas the thymus had lower index LCP and PC group than the $\mathrm{NC}$ group while bursa indexes were lower in the HCP group compared to the $\mathrm{PC}$ group $(\mathrm{P}<$ $0.05)$, the spleen index showed no significant differences between groups $(\mathrm{P}>0.05)$ (See Figure 4). 

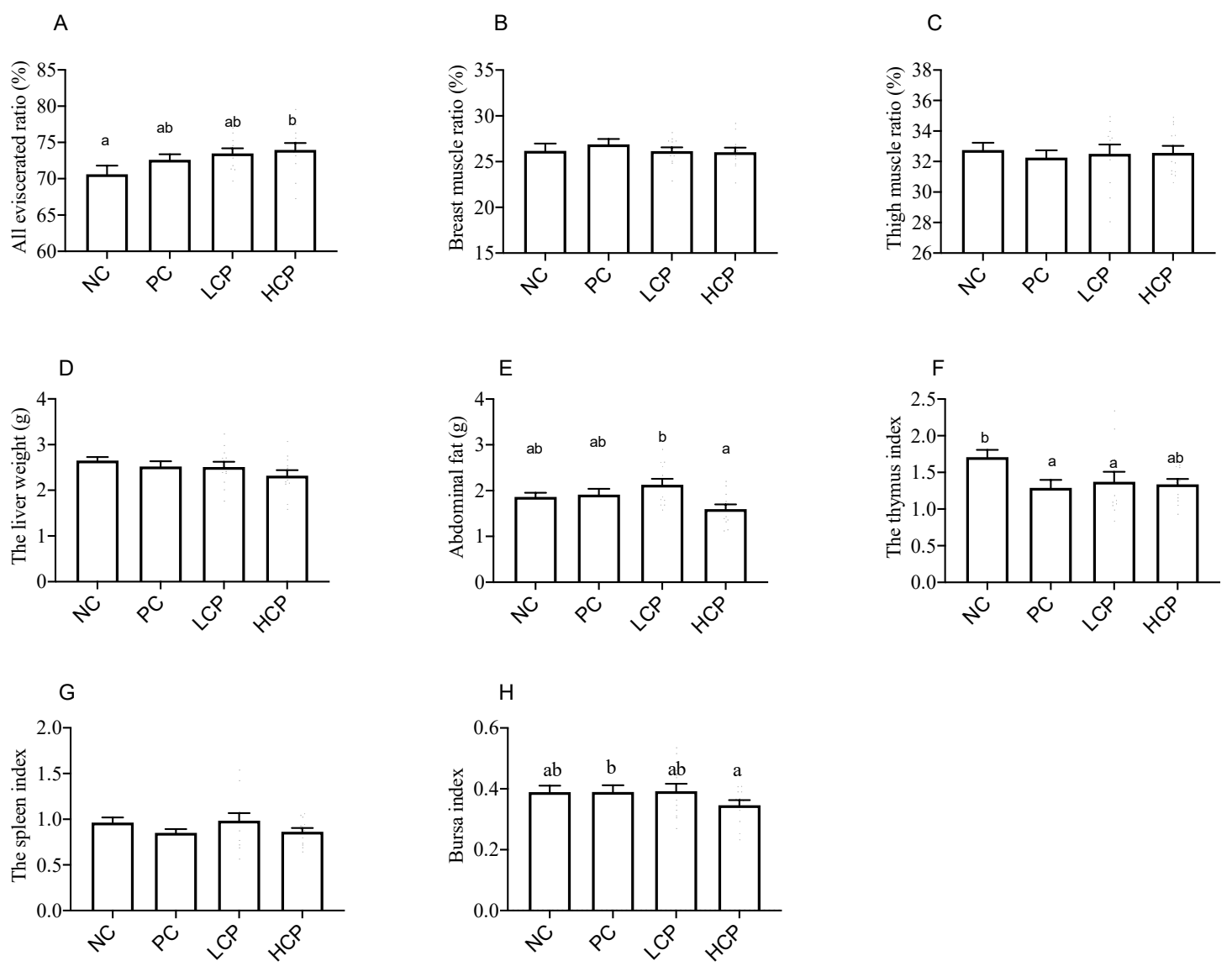

Figure 4. Effect of antibiotic and phenylpyruvate on eviscerated yield, component parts, meat ratios, and immune organs in broiler chicken.

Negative control (NC), Positive control (Antibiotic) (PC), phenylpyruvate (low dose) (LCP), phenylpyruvate (high dose) (HCP).

Values with different superscripts in the same row differ $(P<0.05)$. Different color bar plots showing different groups.

\section{5. pro-inflammatory cytokine}

The gene expression of pro-inflammatory cytokine IL-1 was lower in HCP group than the LCP and PC group whereas HCP group had similar TLR4 levels in relation to the NC group however lower than the PC group (P $<0.05$; Figure 5), whereas IL-10/, IL-4/, IFN-/, and IL-6/ were not different between groups. 

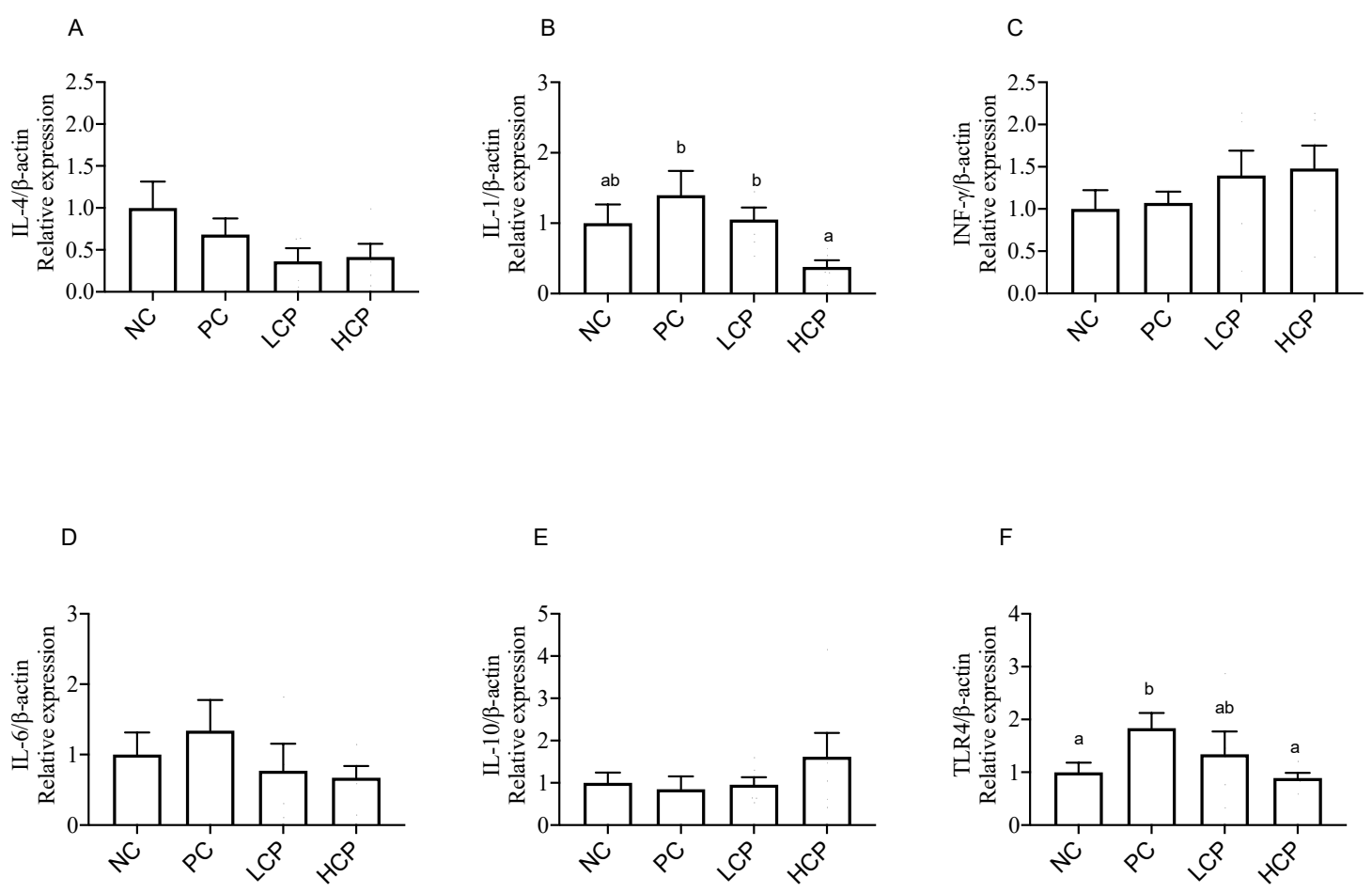

Figure 5. Effect of antibiotic and phenylpyruvate on the relative gene expression of inflammatory cytokines of broiler chickens

Negative control (NC), Positive control (Antibiotic) (PC), phenylpyruvate (low dose) (LCP), phenylpyruvate (high dose) (HCP).

Values with different superscripts in the same row differ $(P<0.05)$. Interleukin 1 beta, Tolllike receptor 4, Interleukin 6, Interleukin 10, Interleukin 4. IL-1 $\beta / \beta$-Actin.

\subsection{Analyses of microbiota composition}

The Shannon analysis was applied to the data to illustrate the variation of the caecal microbiota between the groups (Figure $\underline{6}$ ). The Shannon index - which shows the diversity of species in each community - exhibited a decreasing trend in species richness between the assessed groups (Figure 6). The Shannon index was lower in the LCP and PC group than in the HCP and NC group (Kruskal-Wallis test, $p>0.05$ ), although no differences were observed in the HCP and NC groups.

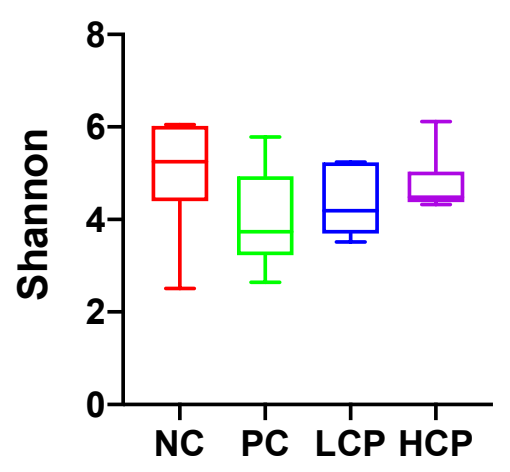

Figure 6. Box-plot comparisons of bacterial diversity and species richness. Bacterial diversity of the different groups using Shannon's alpha index and Species Richness in the 
different groups.Negative control (NC), Positive control (Antibiotic) (PC), phenylpyruvate (low dose) (LCP), phenylpyruvate (high dose) (HCP).

After the analyses of microbiota composition, we found that the dominant phyla of these groups were Firmicutes, Bacteroidata, compylobacteriota, and proteobacteria (Figure 7A). The dominant class was bacilli, bacterioda, clostridia, and negativicutes (Figure $\underline{7} \mathrm{~B})$. The dominant family were lactobacillae, bacterideceae, and veillonellales (Figure 7C). Whereas the dominant genus was lactobacillus, Bacteroides, and Alisptes (Figure 7D). The dominant species included Bacteroides, phelibetes, lactobacillus aviarus, bacteriodes spp millerius (Figure 7E). In different groups, no significant differences were found in the abundance of major phylum Firmicutes and Bacteroidetes, whereas PC group had a lower abundance of Cyanobacteria phylum compared than other groups $(P<0.05$; Figure $\underline{8})$. The class of Vampirivibrionia was also decreased in PC group than the other groups $(P<0.05$; Figure $\underline{8})$, while the abundance of order Veillonellales_selenomonadales higher in HCP group than the other groups $(P<0.05$; Figure $\underline{8})$. The family having Selenomonadaceae were significantly increased in the HCP than the PC group $(P<0.05$; Figure $\underline{8})$ while in family abundance Oscillospiraceae was also higher in NC group than the PC group $(P<0.05$; Figure $\underline{8})$. HCP and LCP groups were also increased the abundance of the genus Megamonas $(P<0.05$; Figure 8). HCP group had increased the abundance of the bacteriodes_plebeius species followed by the LCP group compared with other groups $(P<0.05$; Figure $\underline{8})$.
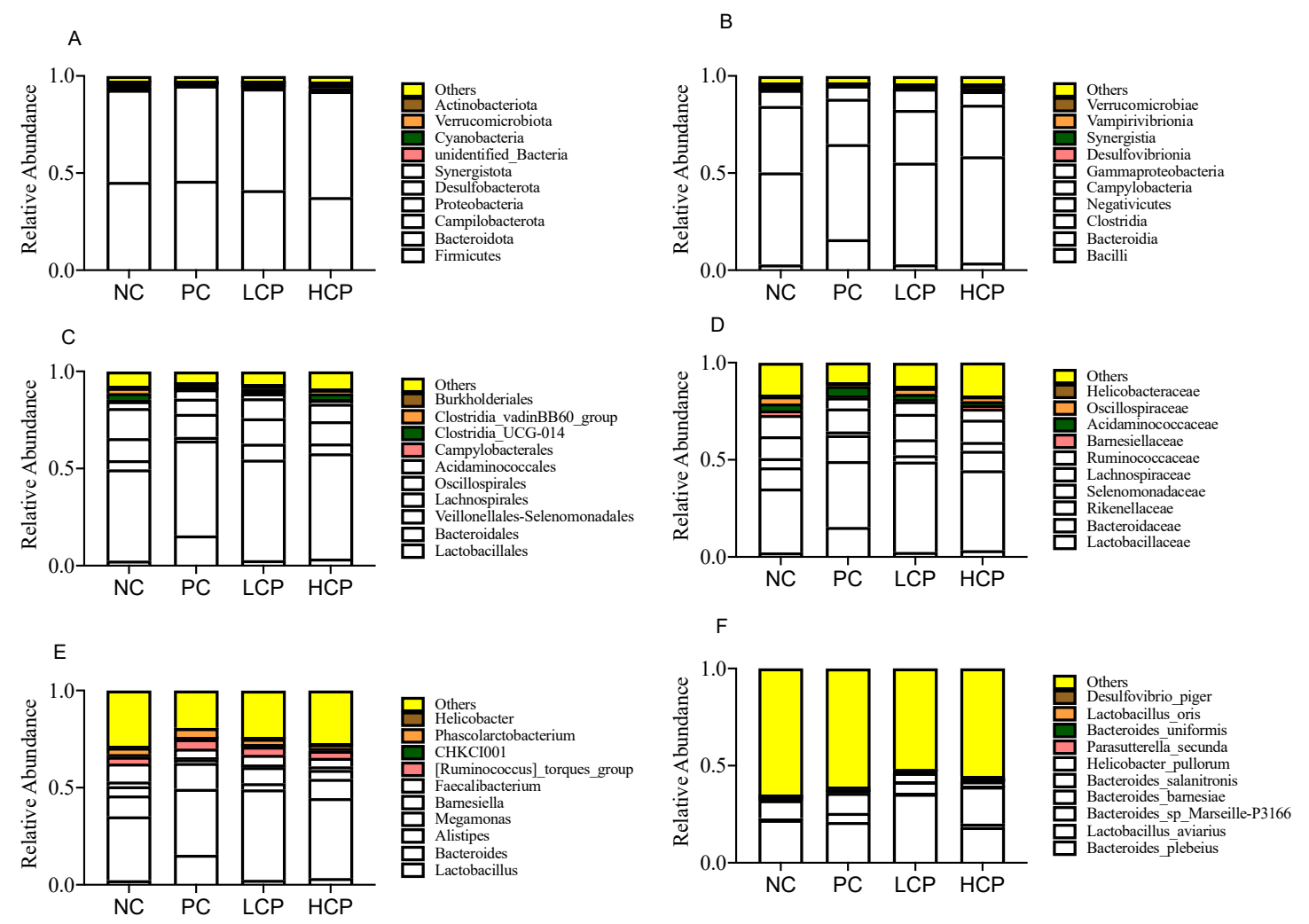

Figure 7. Effect of antibiotic and phenylpyruvate on the Relative Abundance of broiler chickens

Negative control (NC), Positive control (Antibiotic) (PC), phenylpyruvate (low dose) (LCP), phenylpyruvate (high dose) (HCP). 
The microbiome compositions in cecum at phylum, class, family genus, and species-level composition of the caecal microbiome of chicken. A color-coded bar plot shows the average bacterial phylum, class, family, genus, and species distribution in different treatment groups and control groups. Each bar chart represents the relative abundance of each group. Each color represents a specific bacteria phylum (A) a specific bacteria class (B) a specific bacteria order (C) a specific bacteria family (D) a specific bacteria genus (E) and a specific bacteria species $(\mathbf{F})$
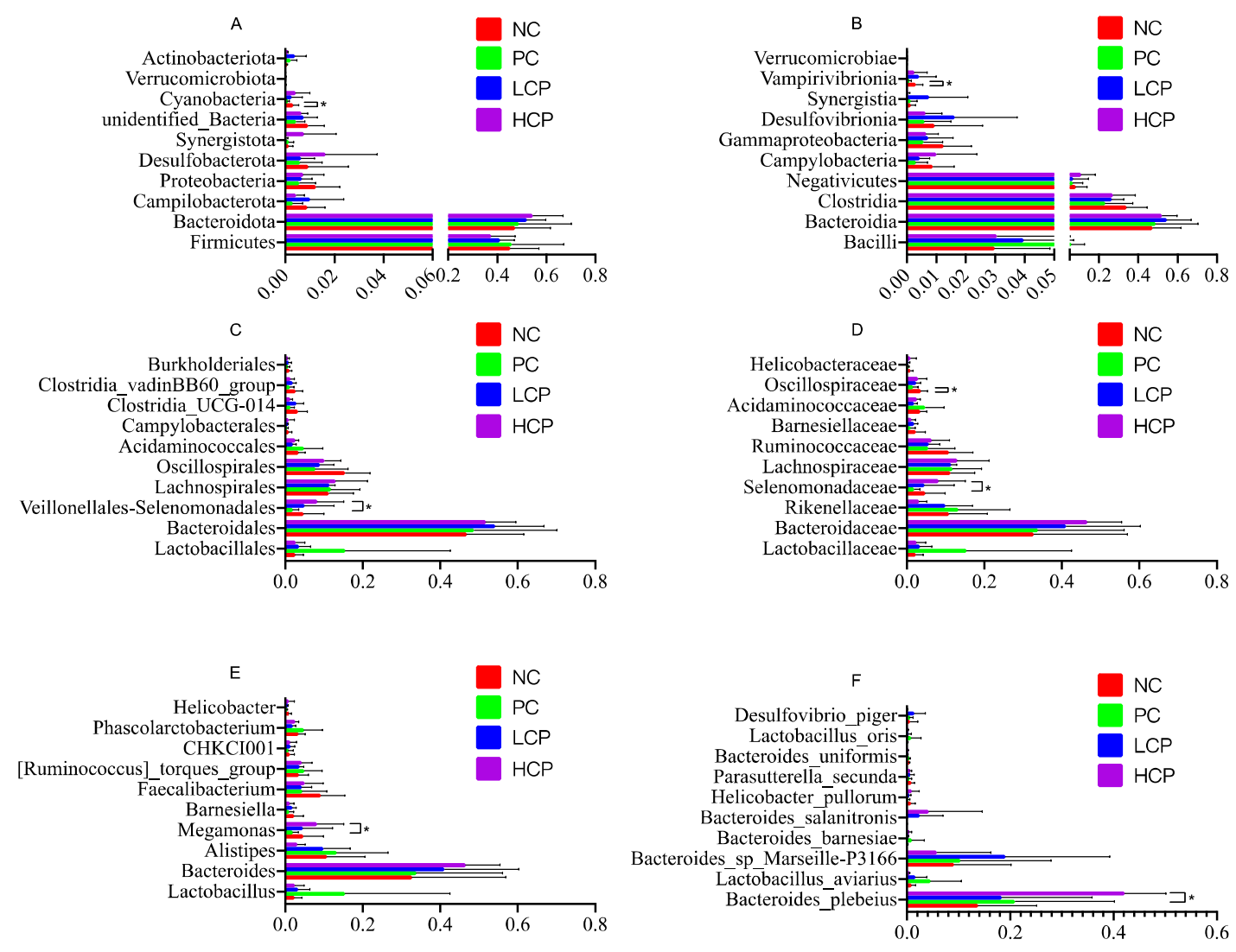

Figure 8. Effect of antibiotic and phenylpyruvate on the H-test bar plot on phylum, class, order, family, genus, and species level of broiler chickens.

Negative control (NC), Positive control (Antibiotic) (PC), phenylpyruvate (low dose) (LCP), phenylpyruvate (high dose) (HCP).

The bacterial clades showing difference at phylum, class, order, family, genus, and species levels in different treatment groups and control group

(A) bacterial clades significantly different at the phylum level in different groups $(\mathrm{P}<0.05$ is showing significant difference).

(B) bacterial clades significantly different at the class level in different groups $(\mathrm{P}<0.05$ is showing significant difference).

(C) bacterial clades significantly different at the order level in different groups $(\mathrm{P}<0.05$ is showing significant difference ).

(D) bacterial clades significantly different at the family level in different groups $(\mathrm{P}<0.05$ is showing significant difference).

(E) bacterial clades significantly different at the genus level in different groups $(\mathrm{P}<0.05$ is showing significant difference). 
(F) bacterial clades significantly different at the species level in different groups $(\mathrm{P}<0.05$ is showing significant difference).

\section{Discussion}

A surge of information regarding the gut microbiota and its impacts on health has been reported over the past decade. The gut microbial community structure is strongly affected by many factors such as diet, age, immunological response, host genotype, antibiotic addition, and pathogen infection [17]. Previous researches have demonstrated that diet has a great influence (estimated at $57 \%$, compared with $12 \%$ for genetic factors) on the gut microbial community structure [18]. The community structure of microbiota can be quickly altered by the changes of dietary components [19]. Phenylalanine and its metabolite phenylpyruvate possesses potential benefits for both humans and animals against numerous diseases, in a previous studies, dietary L-arginine supplementation protected the gut mucosa by improving innate immune responses, intestinal absorption and barrier function [20]. However, little is known regarding how phenylalanine influences the intestinal microbiota in broiler chickens.

Currently, our results suggest that dietary supplements of phenylpyruvate are likely to improve the health status without affecting productive performance, but they did not compare to the effects of antibiotics, which showed better FCR compared to the control group. It is clear that phenylpyruvate can be utilized to improve FCR responses, but this is less efficient than antibiotics, in terms of growth performance but more efficient in improving productive performance.

The metabolite of phenylalanine, phenylpyruvic acid, has been stated in a review by Rose [21] to be capable of promoting growth in the absence of phenylalanine. As far as we are aware, no experimental data were reported. Neither confirmation nor denial of this statement has appeared subsequently-in the literature. Therefore, this metabolite was tested for its ability for improve growth performance in the diet of the growing chickens. Bubl and Butts [22] described that when the basal diet was supplemented with $0.5 \%$ phenylalanine and $0.25 \%$ alanine, poor growth resulted while when the above diet was supplemented with $0.5 \% \mathrm{p}$ hydroxyphenylpyruvic acid, excellent growth was obtained. Apparently sufficient nitrogen is present to allow animation of the compound.

There was no mortality in LCP and the HCP groups during 1-21 days and consistent with studies of Gibson, et al. [23] found decrease in mortality in mycotoxin fed chicken during 121 weeks and may indicate that the Phe supplementation provided protection against ochratoxicosis as a result of the increased dietary Phe. In another study in which $2.4 \% \mathrm{~L}$-alanine was substituted for $2.4 \%$ Phe and decreased mortality (unpublished data), higher mortality for 22-42 days might be due to higher phe requirement of the body because most of studies are only evaluate 1-21 days of chickens.

According to the data, there are significant differences in the carcass characters $(P<$ 0.05). Broilers fed with HCP and LCP diet, resulted in increased eviscerated yield and lower abdominal fat than the control group. Eviscerated yield and abdominal fat rate were essential indicators for evaluating the slaughtering performance of broilers. Maynard, et al. [24] reported that the decrease supplement or deletion of Phe from corn-soybean meal poultry diets caused a negative effect on carcass parts weights, it showing that phenylpyruvate as supplement support optimum growth of fast-growing commercial broilers.

The animal gastrointestinal tract is an important site for the communication of the internal and external environments, and its growth and development status would affect the absorption of nutrients by animals, thereby directly affecting the growth and performance of animals. An increase of villi size is associated with an increase in digestive function for nutrient absorption [25]. Awad, et al. [26] stated that the increase of villi height in the chicken intestine is parallel to the increase of digestive and absorption functions as well as a smooth expression of 
the nutrient transport system throughout the body. Fitasari [27] stated that one of the parameters that can be used to measure the quality of growth is the intestinal morphological structure. Previously chickens are given amino acids metabolites which can improve digestibility in the ileum and can also increase the digestibility of other amino acids such as isoleucine, phenylalanine, valine, aspartic acid, and tyrosine [28].

In the current study, birds fed diets supplemented with HCP had better villus morphology in the ileum than the control group mainly reflecting improved development, as shown by increased villus height and crypt depth. Studies on the effects of phenylalanine or phenylpyruvate on gastrointestinal development in poultry have rarely been reported. The regulation of phenylalanine on pancreatic exocrine has been reported in calves [29]. Other essential amino acids also have effects on the gastrointestinal tract of chickens. Shen, et al. [30] and Zhang, et al. [31] reported the supplementation of young chickens with essential amino acids improved villus development and redox status of the duodenum and ileum. The study by Ao, et al. [32] showed that higher pathogenic bacterial load in the GIT reduced the VH and VH:CD. Higher pathogenic bacterial load was evidenced by the lower serum interleukin IL $\beta$ and TL4 levels in HCP group. Similarly Macelline, et al. [33] found that birds fed with synthetic key essential amino acids showed higher villus height and crypt depth and better immunity as compared with those of broilers fed no synthetic amino acids.

In our study the thymus index was similar in LCP and PC groups but lower than the NC group while bursa indexes were lower in the HCP group than the PC group, the spleen index showed no significant differences between groups. The lymphoid compartments vary in their responses to the inclusion of different feeds in chicken due to the different roles in the immune system. The bursa of Fabricius and spleen are sites of differentiation of B cells and T cells, respectively [34]. Variations in thymus weight could be associated with changes in the lymphoid organs' function. Hence higher thymus index in the NC group might be due to higher exposure of infections or a reduced capacity to maintaining production potential to meet the sanitation challenges [35]. It is important to realise that the lowest weight may not necessarily be linked with lower production of lymphoid cells; so this is essential that also consider other parameters of immunity status [36,37].

In current study at the phylum level Cyanobacteria had a lower concentration in PC than other groups. The ability of cyanobacteria to use amino acids as sole nitrogen sources $[38,39]$ varies greatly between species. Megomonas was found in higher concentrations in HCP than other groups, and previous studies have shown that higher abundances of this genus in poultry have beneficial effects. It was discovered that the Megamonas genus was specific to the $C$. jejuni-suppressive treatment, and Campylobacter-free flocks had high relative abundances of Megamonas [40]. Campylobacter jejuni, the most common cause of bacterial food poisoning, and Megamonas, which can promote the microbial production of short-chain fatty acids (SCFAs) in the chicken intestine [41,42].

Indeed, members of the Oscillospiraceae were previously shown to be increased abundantly due to phytase supplementation [43], whereas in our study family abundance Oscillospiraceae were also higher in NC compared with PC group, on the other hand the family having Selenomonadaceae were significantly increased in the HCP than PC group. family of Selenomonadaceae play an important role in the maintenance of the cell surface structure [44]. Furthermore, characterized by their ability to use a broad range of substrates including the fermentation products of other bacteria [45]. The genus Bacteroides displays a high flexibility to adapt to the nutritional conditions of the intestinal environment [46], being able to use dietary or host-derived glycans according to the nutrient availability [47]. Bacteroides can also incorporate amino acids from outside [48] which could be used to maintain cell structures and as an energy source. In a previous study a species of bacteriodes have been found to utilize leucine, isoleucine and phenylalanine. [49]. This points to a potential capacity of $B$. fragilis (as 
may probably occur with other Bacteroidetes) for regulating BCAA and aromatic amino acids levels in its growth environment. These studies just commented support the interdependence between diet, gut microbiome and host metabolism, and allow to hypothesize that the amino a acids and their metabolites that provide of dietary organic nitrogen sources may be used to modify the metabolic activity of colonic Bacteroides populations by modifying the profile of organic acids formed and enhancing propionate formation in some parts of the large intestine while promoting shifts toward healthier profiles of serum amino acids.

\section{Conclusion}

In conclusion, phenylpyruvate feed additive had the potential to improve the growth performance, intestinal morphology and might be beneficial to some aspects of immunity (regulation of immune genes) possibly due to altered cecal microbial composition however this is less efficient than antibiotics, although more efficient in improving productive performance and gut morphology. Moreover, high dose of phenylpyruvate is more effective than the low dose.

Author Contributions: Conceptualization, M.Z. and W.P.; methodology, Y.Z.; software, P.E.; validation, J.L., H.Z. and G.S.; formal analysis, M.Z.; investigation, Q.J.; resources, P.E.; data curation, H.Z.; writing-original draft preparation, M.Z.; writing-review and editing, G.S.; visualization, W.P.; supervision, G.S.; project administration, Q.J.; funding acquisition, H.Z. All authors have read and agreed to the published version of the manuscript.

Acknowledgments: Guangdong key research and development program (2019B020218001); the local innovative and research teams project of Guangdong province (2019BT02N630).

Conflicts of Interest: The authors declare no conflict of interest.

\section{References:}

1. Mund, M.D.; Khan, U.H.; Tahir, U.; Mustafa, B.-E.-.; Fayyaz, A. Antimicrobial drug residues in poultry products and implications on public health: A review. Int. J. Food Prop. 2017, 20, 1433-1446.

2. Haque, M.; Sarker, S.; Islam, M.; Karim, M.; Kayesh, M.E.H.; Shiddiky, M.J.; Anwer, M.S. Sustainable Antibiotic-Free Broiler Meat Production: Current Trends, Challenges, and Possibilities in a Developing Country Perspective. Biology 2020, 9, 411.

3. Hamid, H.; Zhang, J.; Li, W.; Liu, C.; Li, M.; Zhao, L.; Ji, C.; Ma, Q. Interactions between the cecal microbiota and non-alcoholic steatohepatitis using laying hens as the model. Poult. Sci. 2019, 98, 2509-2521.

4. Kidd, M.; Maynard, C.; Mullenix, G. Progress of amino acid nutrition for diet protein reduction in poultry. J. Anim. Sci. Biotechnol. 2021, 12, 1-9.

5. Sullivan, Å.; Edlund, C.; Nord, C.E. Effect of antimicrobial agents on the ecological balance of human microflora. Lancet Infect. Dis. 2001, 1, 101-114.

6. Ma, N.; Ma, X. Dietary amino acids and the gut-microbiome-immune axis: physiological metabolism and therapeutic prospects. Compr. Rev. Food Sci. F. 2019, $18,221-242$.

7. Ghoreyshi, S.M.; Omri, B.; Chalghoumi, R.; Bouyeh, M.; Seidavi, A.; Dadashbeiki, M.; Lucarini, M.; Durazzo, A.; van den Hoven, R.; Santini, A. Effects of dietary supplementation of L-carnitine and excess lysine-methionine on growth performance, carcass characteristics, and immunity markers of broiler chicken. Animals 2019, 9, 362. 
8. Austic, R. Phiddling with phenylalanine: influence of dietary amino acids on enzymes of phenylalanine metabolism in the chick. In Proceedings of the Cornell Nutrition Conference For Feed Manufacturers, 2009; p. 122.

9. Ross, L.G.; Ross, B. Anaesthetic and sedative techniques for aquatic animals; John Wiley \& Sons: 2009.

10. Arends, R.; Mancera, J.; Munoz, J.; Bonga, S.W.; Flik, G. The stress response of the gilthead sea bream (Sparus aurata L.) to air exposure and confinement. J. Endocrinol. 1999, 163, 149.

11. Cotoia, A.; Scrima, R.; Gefter, J.V.; Piccoli, C.; Cinnella, G.; Dambrosio, M.; Fink, M.P.; Capitanio, N. p-Hydroxyphenylpyruvate, an intermediate of the Phe/Tyr catabolism, improves mitochondrial oxidative metabolism under stressing conditions and prolongs survival in rats subjected to profound hemorrhagic shock. PLoS One 2014, 9, e90917.

12. Salamanca, N.; Giráldez, I.; Morales, E.; de La Rosa, I.; Herrera, M. Phenylalanine and Tyrosine as Feed Additives for Reducing Stress and Enhancing Welfare in Gilthead Seabream and Meagre. Animals 2021, 11, 45.

13. NRC, U. Nutrient requirements of poultry. 1994.

14. Dewar, M.L.; Arnould, J.P.; Dann, P.; Trathan, P.; Groscolas, R.; Smith, S. Interspecific variations in the gastrointestinal microbiota in penguins. Microbiologyopen 2013, 2, 195-204.

15. Xia, J.; Wishart, D.S. Web-based inference of biological patterns, functions and pathways from metabolomic data using MetaboAnalyst. Nat. Protoc. 2011, 6, 743760.

16. Zuidhof, M.; Robinson, F.; Feddes, J.; Hardin, R.; Wilson, J.; McKay, R.; Newcombe, $M$. The effects of nutrient dilution on the well-being and performance of female broiler breeders. Poult. Sci. 1995, 74, 441-456.

17. Luo, Y.; Zhang, L.; Li, H.; Smidt, H.; Wright, A.-D.G.; Zhang, K.; Ding, X.; Zeng, Q.; Bai, S.; Wang, J. Different types of dietary fibers trigger specific alterations in composition and predicted functions of colonic bacterial communities in BALB/c mice. Front. Microbiol. 2017, 8, 966.

18. Tomasello, G.; Tralongo, P.; Damiani, P.; Sinagra, E.; Di Trapani, B.; Zeenny, M.N.; Hussein, I.H.; Jurjus, A.; Leone, A. Dismicrobism in inflammatory bowel disease and colorectal cancer: changes in response of colocytes. World J. Gastroenterol.: WJG 2014, 20, 18121.

19. David, L.A.; Maurice, C.F.; Carmody, R.N.; Gootenberg, D.B.; Button, J.E.; Wolfe, B.E.; Ling, A.V.; Devlin, A.S.; Varma, Y.; Fischbach, M.A. Diet rapidly and reproducibly alters the human gut microbiome. Naure 2014, 505, 559-563.

20. Zhang, B.; Lv, Z.; Li, H.; Guo, S.; Liu, D.; Guo, Y. Dietary l-arginine inhibits intestinal Clostridium perfringens colonisation and attenuates intestinal mucosal injury in broiler chickens. Br. J. Nutr. 2017, 118, 321-332.

21. Rose, W.C. The nutritive significance of the amino acids and certain related compounds. Science 1937, 86, 298-300.

22. Bubl, E.C.; Butts, J.S. The utilization of some phenylpyruvic acids for growth in the rat. J. Biol. Chem. 1949, 180, 839-843.

23. Gibson, R.; Bailey, C.; Kubena, L.; Huff, W.; Harvey, R. Impact of L-Phenylalanine Supplementation on the Performance of Three-Week-Old Broilers Fed Diets Containing Ochratoxin A.: 1. Effects on Body Weight, Feed Conversion, Relative Organ Weight, and Mortality. Poult. Sci. 1990, 69, 414-419. 
24. Maynard, C.; Liu, S.; Lee, J.; Caldas, J.; Diehl, E.; Rochell, S.; Kidd, M. Determining the 4th limiting amino acid in low crude protein diets for male and female cobb mv $\times$ 500 broilers. Br. Poult. Sci. 2020, 61, 695-702.

25. Sun, X.; McElroy, A.; Webb Jr, K.; Sefton, A.; Novak, C. Broiler performance and intestinal alterations when fed drug-free diets. Poult. Sci. 2005, 84, 1294-1302.

26. Awad, W.; Ghareeb, K.; Nitsch, S.; Pasteiner, S.; Abdel-Raheem, S.; Böhm, J. Effects of dietary inclusion of prebiotic, probiotic and synbiotic on the intestinal glucose absorption of broiler chickens. Int. J. Poult. Sci. 2008, 7, 686-691.

27. Fitasari, E. Penggunaan enzim papain dalam pakan terhadap karakteristik usus dan penampilan produksi ayam pedaging. Buana Sains 2011, 12, 7-16.

28. Selle, P.; Ravindran, V.; Ravindran, G.; Bryden, W. Effects of dietary lysine and microbial phytase on growth performance and nutrient utilisation of broiler chickens. Asian-australas. J. Anim. Sci. 2007, 20, 1100-1107.

29. Guo, L.; Tian, H.; Shen, J.; Zheng, C.; Liu, S.; Cao, Y.; Cai, C.; Yao, J. Phenylalanine regulates initiation of digestive enzyme mRNA translation in pancreatic acinar cells and tissue segments in dairy calves. Biosci. Rep. 2018, 38.

30. Shen, Y.; Ferket, P.; Park, I.; Malheiros, R.; Kim, S. Effects of feed grade Lmethionine on intestinal redox status, intestinal development, and growth performance of young chickens compared with conventional DL-methionine. J. Anim. Sci. 2015, 93, 2977-2986.

31. Zhang, Y.; Xu, R.; Min, L.; Ruan, D.; Kim, H.; Hong, Y.; Chen, W.; Wang, S.; Xia, W.; Luo, X. Effects of L-methionine on growth performance, carcass quality, feather traits, and small intestinal morphology of Pekin ducks compared with conventional DL-methionine. Poult. Sci. 2019, 98, 6866-6872.

32. Ao, Z.; Kocher, A.; Choct, M. Effects of dietary additives and early feeding on performance, gut development and immune status of broiler chickens challenged with Clostridium perfringens. Asian-australas. J. Anim. Sci. 2012, 25, 541.

33. Macelline, S.P.; Wickramasuriya, S.S.; Cho, H.M.; Kim, E.; Shin, T.K.; Hong, J.S.; Kim, J.C.; Pluske, J.R.; Choi, H.J.; Hong, Y.G. Broilers fed a low protein diet supplemented with synthetic amino acids maintained growth performance and retained intestinal integrity while reducing nitrogen excretion when raised under poor sanitary conditions. Poult. Sci. 2020, 99, 949-958.

34. Cooper, M.D.; Peterson, R.D.; South, M.A.; Good, R.A. The functions of the thymus system and the bursa system in the chicken. J. Exp. Med. 1966, 123, 75-102.

35. Fasina, Y.; Classen, H.; Garlich, J.; Black, B.; Ferket, P.; Uni, Z.; Olkowski, A. Response of turkey poults to soybean lectin levels typically encountered in commercial diets. 2. Effect on intestinal development and lymphoid organs. Poult. Sci. 2006, 85, 870-877.

36. Kabir, S.L.; Rahman, M.; Rahman, M.; Rahman, M.; Ahmed, S. The dynamics of probiotics on growth performance and immune response in broilers. Int. J. Poult. Sci. 2004, 3, 361-364.

37. Makram, A.; Galal, A.; Fathi, M.; El-Attar, A. Carcass characteristics and immunocompetence parameters of four commercial broiler strain chickens under summer season of Egypt. Int. J. Poult. Sci. 2010, 9, 171-176.

38. Neilson, Z.; Larsson, T. The utilization of organic nitrogen for growth of algae: physiological aspects [cyanobacteria, nitrogen sources, sulphur sources, chlorophyll a]. Physiologia Plantarum (Denmark) 1980.

39. Vaishampayan, A. Amino acid nutrition in the blue-green alga Nostoc muscorum. New Phytologist 1982, 90, 545-549. 
40. Scupham, A.; Jones, J.; Rettedal, E.; Weber, T. Antibiotic manipulation of intestinal microbiota to identify microbes associated with Campylobacter jejuni exclusion in poultry. Appl. Environ. Microbiol. 2010, 76, 8026-8032.

41. Chen, Y.; Ni, J.; Li, H. Effect of green tea and mulberry leaf powders on the gut microbiota of chicken. BMC Vet. Res. 2019, 15, 1-6.

42. Sergeant, M.J.; Constantinidou, C.; Cogan, T.A.; Bedford, M.R.; Penn, C.W.; Pallen, M.J. Extensive microbial and functional diversity within the chicken cecal microbiome. PloS one 2014, 9, e91941.

43. Klinsoda, J.; Vötterl, J.; Koger, S.; Metzler-Zebeli, B.U. Dietary phytase-and lactic acid-treated cereals caused greater taxonomic adaptations than functional adaptations in the cecal metagenome of growing pigs. Appl. Environ. Microbiol. 2020, 87, e02240-02220.

44. Kojima, S.; Ko, K.-C.; Takatsuka, Y.; Abe, N.; Kaneko, J.; Itoh, Y.; Kamio, Y.

Cadaverine covalently linked to peptidoglycan is required for interaction between the peptidoglycan and the periplasm-exposed S-layer-homologous domain of major outer membrane protein Mep45 in Selenomonas ruminantium. J. Bacteriol. 2010, 192, 5953-5961.

45. Rasmussen, M.A. Isolation and characterization of Selenomonas ruminantium strains capable of 2-deoxyribose utilization. Appl. Environ. Microbiol. 1993, 59, 2077-2081.

46. Comstock, L.E.; Coyne, M.J. Bacteroides thetaiotaomicron: a dynamic, niche-adapted human symbiont. Bioessays 2003, 25, 926-929.

47. Sonnenburg, J.L.; Xu, J.; Leip, D.D.; Chen, C.-H.; Westover, B.P.; Weatherford, J.; Buhler, J.D.; Gordon, J.I. Glycan foraging in vivo by an intestine-adapted bacterial symbiont. Science 2005, 307, 1955-1959.

48. Smith, E.A.; Macfarlane, G.T. Enumeration of amino acid fermenting bacteria in the human large intestine: effects of $\mathrm{pH}$ and starch on peptide metabolism and dissimilation of amino acids. FEMS microbiology ecology 1998, 25, 355-368.

49. Rios-Covian, D.; Sánchez, B.; Salazar, N.; Martínez, N.; Redruello, B.; Gueimonde, M.; de Los Reyes-Gavilán, C.G. Different metabolic features of Bacteroides fragilis growing in the presence of glucose and exopolysaccharides of bifidobacteria. Front. Microbiol. 2015, 6, 825 . 